\title{
Global Solutions to the Spherically Symmetric Compressible Navier-Stokes Equations with Density-Dependent Viscosity and Discontinuous Initial Data
}

\author{
Ruxu Lian, ${ }^{1,2}$ Jianwei Yang, ${ }^{1}$ and Jian Liu ${ }^{3}$ \\ ${ }^{1}$ College of Mathematics and Information Science, North China University of Water Resources and Electric Power, \\ Zhengzhou 450011, China \\ ${ }^{2}$ Institute of Atmospheric Physics, Chinese Academy of Sciences, Beijing 100029, China \\ ${ }^{3}$ College of Teacher Education, Quzhou University, Quzhou 324000, China \\ Correspondence should be addressed to Ruxu Lian; ruxu.lian.math@gmail.com
}

Received 29 September 2013; Accepted 8 April 2014; Published 4 May 2014

Academic Editor: Adem Kılıçman

Copyright (C) 2014 Ruxu Lian et al. This is an open access article distributed under the Creative Commons Attribution License, which permits unrestricted use, distribution, and reproduction in any medium, provided the original work is properly cited.

We consider the initial boundary value problem for the spherically symmetric isentropic compressible Navier-Stokes equations with density-dependent viscosity coefficients and discontinuous initial data in this paper. For piecewise regular initial density with bounded jump discontinuity, we show that there exists a unique global piecewise regular solution. In particular, the jump of density decays exponentially in time and the piecewise regular solution tends to the equilibrium state exponentially as $t \rightarrow+\infty$.

\section{Introduction}

The isentropic compressible Navier-Stokes equations with density-dependent viscosity coefficients read as follows:

$$
\begin{aligned}
& \rho_{t}+\operatorname{div}(\rho \mathbf{U})=0, \\
& \begin{aligned}
(\rho \mathbf{U})_{t} & +\operatorname{div}(\rho \mathbf{U} \otimes \mathbf{U})+\nabla P(\rho) \\
& -\operatorname{div}(h(\rho) D(\mathbf{U}))-\nabla(g(\rho) \operatorname{div} \mathbf{U})=0,
\end{aligned}
\end{aligned}
$$

where $t \in(0,+\infty)$ is the time and $\mathbf{x} \in R^{N}, N$ is the spatial coordinate, and $\rho>0$ and $u$ denote the density and velocity, respectively. Pressure function is taken as $P(\rho)=\rho^{\gamma}$ with $\gamma>$ 1 , and

$$
D(\mathbf{U})=\frac{\nabla(\mathbf{U})+{ }^{\mathbb{T}} \nabla(\mathbf{U})}{2}
$$

is the strain tensor and $h(\rho), g(\rho)$ are the Lamé viscosity coefficients satisfying

$$
h(\rho)>0, \quad h(\rho)+N g(\rho) \geq 0 .
$$

There is huge literature on the studies of the global existence of weak solutions and dynamical behaviors of jump discontinuity for the compressible Navier-Stokes equations with discontinuous initial data; for instance, if the viscosity coefficients $h(\rho)$ and $g(\rho)$ are both constants, for the case of one space dimension, Hoff investigated the global existence of discontinuous solutions of the Navier-Stokes equations [1-3]. Hoff derived the construction of global spherically symmetric weak solutions of compressible Navier-Stokes equations for isothermal flow with large and discontinuous initial data [4]; therein, Hoff also showed that the discontinuities persist for all time, convecting along particle trajectories, and decaying at a rate inversely proportional to the viscosity coefficient. Hoff also obtained the global existence theorems for the multidimensional Navier-Stokes equations of isothermal compressible flows with the polytropic equation of state $p(\rho)=\rho^{\gamma}(\gamma \geq 1)[5,6]$. The global existence of weak solutions was proved for the Navier-Stokes equations for compressible, heat-conducting flow in one space dimension with large, discontinuous initial data by Chen et al. in [7]. Hoff showed the global existence of weak solutions of the Navier-Stokes equations for compressible, heat-conducting 
fluids in two and three space dimensions when the initial data may be discontinuous across a hypersurface of $R^{n}$ [8]. The global existence of solutions of the Navier-Stokes equations for compressible, barotropic flow in two space dimensions which exhibit convecting singularity curves, was proved by Hoff in [9].

If the viscosity coefficients $h(\rho)=\rho^{\alpha}, g(\rho)=0$, for the case of one space dimension, Fang and Zhang proved the global existence of unique piecewise smooth solution to the free boundary value problem for (1) with $0<\alpha<1$, where the initial density is piecewise smooth with possibly large jump discontinuities [10]. Lian et al. addressed the initial boundary value problem for (1) with $0<\alpha \leq 1$ subject to piecewise regular initial data with initial vacuum state included [11], where they obtained the global existence of unique piecewise regular solution and the finite time vanishing of vacuum state. In particular, they got that the jump discontinuity of density decays exponentially but never vanishes in any finite time and the piecewise regular solution tends to the equilibrium state exponentially as $t \rightarrow+\infty$.

In this present paper, we consider the initial boundary value problem for the spherically symmetric isentropic compressible Navier-Stokes equations with density-dependent viscosity coefficients and discontinuous initial data and focus on the regularities and dynamical behaviors of global weak solution and so forth. As $\gamma>1$, we show that the initial boundary value problem with piecewise regular initial data admits a unique global piecewise regular solution, where the discontinuity in piecewise regular initial density is bounded. In particular, the jump discontinuity of density decays exponentially and the piecewise regular solution tends to the equilibrium state exponentially as $t \rightarrow+\infty$.

There are also many significant progresses achieved recently on the compressible Navier-Stokes equations with density-dependent viscosity coefficients. For instance, the mathematical derivations are obtained in the simulation of flow surface in shallow region $[12,13]$. The prototype model is the viscous Saint-Venant equation (corresponding to (1) with $P(\rho)=\rho^{2}, h(\rho)=\rho$, and $\left.g(\rho)=0\right)$. Many authors considered the well-posedness of solutions to the free boundary value problem with initial finite mass and the flow density being connected with the infinite vacuum either continuously or via jump discontinuity; refer to [14-22] and references therein. The global existence of classical solutions is shown by Mellet and Vasseur in [23]. The qualitative behaviors of global solutions and dynamical asymptotics of vacuum states are also made, such as the finite time vanishing of finite vacuum or asymptotical formation of vacuum in long time, the dynamical behaviors of vacuum boundary, the long time convergence to rarefaction wave with vacuum, and the stability of shock profile with large shock strength; refer to [24-26] and references therein.

The rest part of the paper is arranged as follows. In Section 2, the main results about the existence and dynamical behaviors of global piecewise regular solution for compressible Navier-Stokes equations are stated. Then, some important a priori estimates will be given in Section 3. Finally, the theorem is proved in Section 4.

\section{Notations and Main Results}

We will take $h(\rho)=\rho$ and $g(\rho)=0$ and $D(\mathbf{U})=\nabla \mathbf{U}$ in (1) for simplicity. The isentropic compressible Navier-Stokes equations read as

$$
\begin{aligned}
& \rho_{t}+\operatorname{div}(\rho \mathbf{U})=0, \\
& (\rho \mathbf{U})_{t}+\operatorname{div}(\rho \mathbf{U} \otimes \mathbf{U})+\nabla \rho^{\gamma}-\operatorname{div}(\rho \nabla \mathbf{U})=0,
\end{aligned}
$$

with the initial data and boundary condition

$$
\begin{aligned}
& (\rho, \mathbf{U})(\mathbf{x}, 0)=\left(\rho_{0}, \mathbf{U}_{0}\right)(\mathbf{x}), \quad \mathbf{x} \in \Omega, \\
& \mathbf{U}(\mathbf{x}, t)=0, \quad \mathbf{x} \in \partial \Omega, t \in[0, T],
\end{aligned}
$$

where $\Omega:=\left\{\mathbf{x}\left|0<r_{-} \leq\right| \mathbf{x} \mid \leq r_{+}\right\}$and $r_{-}$and $r_{+}$are constants.

We will investigate the spherically symmetric solutions of the system (4) in a spherically symmetric domain $\Omega$ and denote that

$$
|\mathbf{x}|=r, \quad \rho(\mathbf{x}, t)=\rho(r, t), \quad \mathbf{U}(\mathbf{x}, t)=u(r, t) \frac{\mathbf{x}}{r},
$$

which gives the following system for $r>0$ :

$$
\begin{aligned}
& \rho_{t}+(\rho u)_{r}+\frac{2 \rho u}{r}=0, \\
& (\rho u)_{t}+\left(\rho u^{2}+\rho^{\gamma}\right)_{r}+\frac{2 \rho u^{2}}{r}-\left(\rho u_{r}\right)_{r}-\rho\left(\frac{2 u}{r}\right)_{r}=0,
\end{aligned}
$$

and the initial data and boundary condition become

$$
\begin{aligned}
& (\rho, u)(r, 0)=\left(\rho_{0}, u_{0}\right)(r), \quad r \in\left[r_{-}, r_{+}\right], \\
& u\left(r_{-}, t\right)=u\left(r_{+}, t\right)=0, \quad t \in[0, T] .
\end{aligned}
$$

For simplicity, we consider the initial data with one discontinuous point $\xi_{0} \in\left(r_{-}, r_{+}\right)$; namely,

$$
\begin{aligned}
& r^{2} \rho_{0} \in L^{1} \cap W^{1, \infty}\left(\left[r_{-}, \xi_{0}\right) \cup\left(\xi_{0}, r_{+}\right]\right), \\
& \underset{\mathbf{x} \in\left[r_{-} \xi_{0}\right) \cup\left(\xi_{0}, r_{+}\right]}{\rho_{0}>\rho_{-}>0,} \\
& \rho_{0}\left(\xi_{0}-0\right) \neq \rho_{0}\left(\xi_{0}+0\right), \\
& \left|\rho_{0}\left(\xi_{0}+0\right)-\rho_{0}\left(\xi_{0}-0\right)\right|<\delta, \\
& r^{2} u_{0} \in H^{2}\left(\left[r_{-}, \xi_{0}\right) \cup\left(\xi_{0}, r_{+}\right]\right),
\end{aligned}
$$

where $\rho_{-}$and $\delta>0$ are positive constants, and $\delta>0$ is bounded.

Then, we can give the main results as follows.

Theorem 1. Let $\gamma>1$. Assume that the initial data satisfies (9). Then, there exist two positive constants $\rho_{*}, \rho^{*}$ and a unique 
global piecewise regular solution $(\rho, u)$ to the initial boundary value problem (7)-(8), namely, satisfying

$$
\begin{aligned}
& 0<\rho_{*} \leq \rho(r, t) \leq \rho^{*}, \\
& (r, t) \in\left[r_{-}, \xi(t)\right) \cup\left(\xi(t), r_{+}\right] \times[0, T], \\
& \rho \in L^{\infty}\left([0, T] ; H^{1}\left(\left[r_{-}, \xi(t)\right) \cup\left(\xi(t), r_{+}\right]\right)\right), \\
& u \in L^{\infty}\left([0, T] ; H^{1}\left(\left[r_{-}, \xi(t)\right) \cup\left(\xi(t), r_{+}\right]\right)\right), \\
& u_{r} \in L^{2}\left([0, T] ; H^{1}\left(\left[r_{-}, \xi(t)\right) \cup\left(\xi(t), r_{+}\right]\right)\right),
\end{aligned}
$$

where $r=\xi(t)$ is a curve defined by

$$
\frac{d \xi(t)}{d t}=u(\xi(t), t), \quad \xi(0)=\xi_{0}, \quad t>0
$$

along which the Rankine-Hugoniot conditions hold

$$
\begin{aligned}
& {[u(\xi(t), t)]=0,} \\
& {\left[\rho^{\gamma}(\xi(t), t)\right]=\left[\left(\rho u_{r}+\frac{2 \rho u}{r}\right)(\xi(t), t)\right],}
\end{aligned}
$$

where $[f(\xi(t), t)]:=f(\xi(t)+0, t)-f(\xi(t)-0, t)$, and along the discontinuity $r=\xi(t)$ the jump decays exponentially

$$
\left|\left[\rho_{0}\left(\xi_{0}\right)\right]\right| e^{-C_{0} t} \leq|[\rho(\xi(t), t)]| \leq\left|\left[\rho_{0}\left(\xi_{0}\right)\right]\right| e^{-C_{1} t}
$$

and furthermore, the solution $(\rho, u)$ tends to the equilibrium state exponentially

$$
\|(\rho-\bar{\rho}, u)(\cdot, t)\|_{L^{\infty}\left(\left[r_{-}, \xi(t)\right) \cup\left(\xi(t), r_{+}\right]\right)} \leq C_{2} e^{-C_{3} t},
$$

where $C_{0}, C_{1}, C_{2}$, and $C_{3}$ are positive constants independent of time and $\bar{\rho}=1 /\left(r_{+}-r_{-}\right) f_{r_{-}}^{r_{+}} \rho(r, t) r^{2} d r$, where $f_{r_{-}}^{r_{+}}:=$ $\int_{r_{-}}^{\xi(t)}+\int_{\xi(t)}^{r_{+}}$.

Remark 2. Theorem 1 holds for the Saint-Venant model for shallow water; that is, $\gamma=2$.

Remark 3. For the piecewise regular initial data, Hoff [4] has proved that there is a (piecewise regular) weak solution to the initial boundary value problem (7)-(8) in terms of the difference scheme. In addition, there is a curve $r=$ $\xi(t)$ starting from $\xi(0)=\xi_{0}$, such that the density is discontinuous cross the curve $r=\xi(t)$, and the RankineHugoniot conditions hold

$$
\begin{aligned}
& {[u(\xi(t), t)]=0,} \\
& {\left[\rho^{\gamma}(\xi(t), t)\right]=\left[\left(\rho u_{r}+\frac{2 \rho u}{r}\right)(\xi(t), t)\right] .}
\end{aligned}
$$

To extend the local solution globally in time, we have to prove the uniformly a priori estimates.

\section{The a Priori Estimates}

According to the analysis made in [27], there is a curve $r=$ $\xi(t)$ defined by

$$
\frac{d \xi(t)}{d t}=u(\xi(t), t), \quad \xi(0)=\xi_{0}, \quad t>0
$$

along which the Rankine-Hugoniot conditions hold

$$
\begin{aligned}
& {[u(\xi(t), t)]=0,} \\
& {\left[\rho^{\gamma}(\xi(t), t)\right]=\left[\left(\rho u_{r}+\frac{2 \rho u}{r}\right)(\xi(t), t)\right],}
\end{aligned}
$$

where $[f(\xi(t), t)]:=f(\xi(t)+0, t)-f(\xi(t)-0, t)$. It is convenient to make use of the Lagrange coordinates so as to establish the uniformly a priori estimates and take the Lagrange coordinates transform

$$
x=\int_{r_{-}}^{r} \rho(r, t) r^{2} d r, \quad \tau=t .
$$

By (18) and the conservation of mass for $(\rho, u)$

$$
\int_{r_{-}}^{r_{+}} \rho(r, t) r^{2} d r=\int_{r_{-}}^{r_{+}} \rho_{0}(r) r^{2} d r:=1,
$$

the Lagrange coordinates transform (18) maps $(r, t) \in$ $\left[r_{-}, r_{+}\right] \times R^{+}$into $(x, \tau) \in[0,1] \times R^{+}$. The curve $r=\xi(t)$ in Eulerian coordinates is changed to a line $x=x_{0}$ in Lagrangian coordinates, where

$$
x_{0}=\int_{r_{-}}^{\xi(t)} \rho(r, t) r^{2} d r=\int_{r_{-}}^{\xi_{0}} \rho_{0}(r) r^{2} d r \in(0,1) \text {, }
$$

and the jump conditions become

$$
\left[u\left(x_{0}, \tau\right)\right]=0, \quad\left[\rho^{\gamma}\left(x_{0}, \tau\right)\right]=\left[\rho^{2}\left(r^{2} u\right)_{x}\left(x_{0}, \tau\right)\right]
$$

The relations between Lagrangian and Eulerian coordinates are satisfied as

$$
\frac{\partial x}{\partial r}=\rho r^{2}, \quad \frac{\partial x}{\partial t}=-\rho u r^{2}
$$

and the initial boundary value problem (7)-(8) is reformulated to

$$
\begin{aligned}
& \rho_{\tau}+\rho^{2}\left(r^{2} u\right)_{x}=0, \\
& r^{-2} u_{\tau}+\left(\rho^{\gamma}\right)_{x}=\left(\rho^{2}\left(r^{2} u\right)_{x}\right)_{x}-\frac{2 \rho_{x} u}{r}, \\
& (\rho, u)(x, 0)=\left(\rho_{0}, u_{0}\right)(x), \quad x \in[0,1], \\
& u(0, t)=u(1, t)=0, \quad \tau \in[0,+\infty),
\end{aligned}
$$


where the initial data satisfies

$$
\begin{aligned}
& \rho_{0} \in L^{1}([0,1]) \cap W^{1, \infty}\left(\left[0, x_{0}\right) \cup\left(x_{0}, 1\right]\right), \\
& \inf _{x \in\left[0, x_{0}\right) \cup\left(x_{0}, 1\right]} \rho_{0}>\rho_{-}>0, \\
& \rho_{0}\left(x_{0}-0\right) \neq \rho_{0}\left(x_{0}+0\right), \\
& \left|\rho_{0}\left(x_{0}+0\right)-\rho_{0}\left(x_{0}-0\right)\right|<\delta, \\
& \frac{1}{\sqrt{r^{2} \rho_{0}}}\left(r^{2} u_{0}\right) \in L^{2}\left(\left[0, x_{0}\right) \cup\left(x_{0}, 1\right]\right), \\
& \sqrt{r^{2} \rho_{0}}\left(r^{2} u_{0}\right)_{x} \in L^{2}\left(\left[0, x_{0}\right) \cup\left(x_{0}, 1\right]\right), \\
& \frac{1}{\sqrt{r^{2} \rho_{0}}}\left(r^{2} \rho_{0}\left(r^{2} \rho_{0}\left(r^{2} u_{0}\right)_{x}\right)\right) \in L^{2}\left(\left[0, x_{0}\right) \cup\left(x_{0}, 1\right]\right) .
\end{aligned}
$$

Next, we will deduce the a priori estimates for the solution $(\rho, u)$ to the initial boundary value problem (23). To prove the a priori estimates, we assume a priori that there are constants $\rho_{ \pm}>0$ such that

$$
\begin{aligned}
& 0<\rho_{-} \leq \rho(x, \tau) \leq \rho_{+}, \\
& (x, \tau) \in\left(\left[0, x_{0}\right) \cup\left(x_{0}, 1\right]\right) \times[0, T] \\
& T>0 .
\end{aligned}
$$

Lemma 4. Let $T>0$. Under the conditions in Theorem 1, it holds for any solution $(\rho, u)$ to the initial boundary value problem (23) that

$$
\begin{gathered}
f_{0}^{1}\left(\frac{1}{4} u^{2}+\frac{1}{\gamma-1} \rho^{\gamma-1}\right) d x+\int_{0}^{\tau} f_{0}^{1}\left(\frac{2 u^{2}}{r^{2}}+\frac{1}{2} \rho^{2} u_{x}^{2} r^{4}\right) d x d s \\
\leq f_{0}^{1}\left(\frac{1}{2} u_{0}^{2}+\frac{1}{\gamma-1} \rho_{0}^{\gamma-1}\right) d x, \quad \tau \in[0, T],
\end{gathered}
$$

where $f_{0}^{1}:=\int_{0}^{x_{0}}+\int_{x_{0}}^{1}$.

Proof. Multiplying (23) 2 by $r^{2} u$ and integrating the result with respect to $x$ over $[0,1]$, it holds from $(21)$ and $(23)_{1}$ that

$$
\begin{gathered}
\frac{d}{d \tau} f_{0}^{1}\left(\frac{1}{2} u^{2}+\frac{1}{\gamma-1} \rho^{\gamma-1}\right) d x+\int_{0}^{1} \rho^{2}\left(r^{2} u\right)_{x}^{2} d x \\
\quad=2 f_{0}^{1} \rho\left(r u^{2}\right)_{x} d x+\left.2[\rho]\left(r u^{2}\right)\right|_{x=x_{0}},
\end{gathered}
$$

and integrating (27) with respect to $\tau$, we have

$$
\begin{gathered}
f_{0}^{1}\left(\frac{1}{2} u^{2}+\frac{1}{\gamma-1} \rho^{\gamma-1}\right) d x+\int_{0}^{\tau} f_{0}^{1}\left(\frac{2 u^{2}}{r^{2}}+\rho^{2} u_{x}^{2} r^{4}\right) d x d s \\
=f_{0}^{1}\left(\frac{1}{2} u_{0}^{2}+\frac{1}{\gamma-1} \rho_{0}^{\gamma-1}\right) d x+\left.\int_{0}^{\tau} 2[\rho]\left(r u^{2}\right)\right|_{x=x_{0}} d s .
\end{gathered}
$$

Using (21) and (23) $)_{1}$, we deduce

$$
[\rho]_{\tau}+\left[\rho^{\gamma}\right]=0
$$

which gives

$$
[\rho]=\left[\rho_{0}\right] \exp \left(-\int_{0}^{\tau} \frac{\left[\rho^{\gamma}\right]}{[\rho]} d s\right) .
$$

It holds from (25) that

$$
\gamma \rho_{-}^{\gamma-1} \leq \frac{\left[\rho^{\gamma}\right]}{[\rho]} \leq \gamma \rho_{+}^{\gamma-1} .
$$

From (24), (30), and (31), we have

$$
\begin{aligned}
\left|\int_{0}^{\tau} 2[\rho]\left(r u^{2}\right)\right|_{x=x_{0}} d s \mid \\
\leq \bar{C}\left(1+\rho_{-}^{-1}\right) \int_{0}^{\tau}\left|\left[\rho_{0}\right]\right| e^{-\gamma \rho_{-}^{\gamma-1} s}\left(\int_{0}^{1} u^{2} d x+f_{0}^{1} u_{x}^{2} d x\right) d s \\
\leq \bar{C}\left(1+\rho_{-}^{-1}\right)\left|\left[\rho_{0}\right]\right| \sup _{\tau \in[0, T]} f_{0}^{1} u^{2} d x \int_{0}^{\tau} e^{-\gamma \rho_{-}^{\gamma-1} s} d s \\
\quad+\bar{C}\left(1+\rho_{-}^{-1}\right)\left|\left[\rho_{0}\right]\right| \rho_{-}^{-2} r_{-}^{-4} \int_{0}^{\tau} f_{0}^{1} \rho^{2} u_{x}^{2} r^{4} d x d s \\
\leq \frac{1}{4} \sup _{\tau \in[0, T]} f_{0}^{1} u^{2} d x+\frac{1}{2} \int_{0}^{\tau} f_{0}^{1} \rho^{2} u_{x}^{2} r^{4} d x d s,
\end{aligned}
$$

where $\bar{C}$ is a positive constant independent of time and we assume that

$$
\begin{aligned}
0 & <\left|\left[\rho_{0}\right]\right| \\
& \leq \min \left\{\frac{\gamma}{4 \bar{C}\left(1+\rho_{-}^{-1}\right)} \rho_{-}^{\gamma-1}, \frac{1}{2 \bar{C}\left(1+\rho_{-}^{-1}\right)} \rho_{-}^{2} r_{-}^{4}\right\}:=M_{1} .
\end{aligned}
$$

From (28) and (32), Lemma 4 can be proved.

Lemma 5. Let $T>0$. Under the conditions in Theorem 1, it holds for any solution $(\rho, u)$ to the initial boundary value problem (23) that

$$
\begin{aligned}
\frac{1}{2} f_{0}^{1}( & \left.u+r^{2} \rho_{x}\right)^{2} d x+\frac{1}{\gamma-1} \int_{0}^{1} \rho^{\gamma-1} d x \\
& +\gamma \int_{0}^{\tau} f_{0}^{1} \rho^{\gamma-1} \rho_{x}^{2} r^{4} d x d s \\
\leq & \frac{1}{2} \int_{0}^{1}\left(u_{0}+r^{2} \rho_{0 x}\right)^{2} d x \\
& +\frac{1}{\gamma-1} \int_{0}^{1} \rho_{0}^{\gamma-1} d x+C, \quad \tau \in[0, T],
\end{aligned}
$$

where $C$ is a positive constant independent of time. 
Proof. Differentiating $(23)_{1}$ with respect to $x$, we get

$$
\rho_{x \tau}+\left(\rho^{2}\left(r^{2} u\right)_{x}\right)_{x}=0 .
$$

Summing (35) and (23) 2 , it holds

$$
\left(r^{-2} u+\rho_{x}\right)_{\tau}+\left(\rho^{\gamma}\right)_{x}=\left(r^{-2}\right)_{\tau} u-\frac{2 \rho_{x} u}{r} .
$$

Note that

$$
r^{3}(x, \tau)=r_{-}^{3}+3 \int_{0}^{x} \frac{1}{\rho(z, \tau)} d z
$$

and so

$$
\begin{aligned}
\frac{\partial r}{\partial \tau} & =\frac{1}{r^{2}} \int_{0}^{x}\left(\frac{1}{\rho}\right)_{t}(z, t) d z \\
& =\frac{1}{r^{2}} \int_{0}^{x}\left(r^{2} u\right)_{z}(z, t) d z=u(x, \tau)
\end{aligned}
$$

which together with (36) implies

$$
\left(r^{-2} u+\rho_{x}\right)_{\tau}+\left(\rho^{\gamma}\right)_{x}=-2 r^{-3} u^{2}-\frac{2 \rho_{x} u}{r} .
$$

Multiplying (39) by $\left(u+r^{2} \rho_{x}\right) r^{2}$ and integrating the result with respect to $x$ and $\tau$, it holds that

$$
\begin{aligned}
\frac{1}{2} f_{0}^{1}( & \left(u+r^{2} \rho_{x}\right)^{2} d x+\frac{1}{\gamma-1} f_{0}^{1} \rho^{\gamma-1} d x \\
& +\gamma \int_{0}^{\tau} f_{0}^{1} \rho^{\gamma-1} \rho_{x}^{2} r^{4} d x d s \\
= & \frac{1}{2} f_{0}^{1}\left(u_{0}+r^{2} \rho_{0 x}\right)^{2} d x+\frac{1}{\gamma-1} \int_{0}^{1} \rho_{0}^{\gamma-1} d x \\
& +\left.\int_{0}^{\tau}\left[\rho^{\gamma}\right]\left(r^{2} u\right)\right|_{x=x_{0}} d s .
\end{aligned}
$$

From (24), (26), (30), and (31), we have that

$$
\begin{aligned}
& \left|\int_{0}^{\tau}\left[\rho^{\gamma}\right]\left(r^{2} u\right)\right|_{x=x_{0}} d s \mid \\
& \quad \leq \widehat{C}\left(1+\rho_{-}^{-2}\right) \int_{0}^{\tau} \rho_{+}^{\gamma-1}|[\rho]|\left(\int_{0}^{1} u^{2} d x+\int_{0}^{1} u_{x}^{2} d x\right)^{1 / 2} d s \\
& \quad \leq \widehat{C}\left(1+\rho_{-}^{-2}\right) \int_{0}^{\tau}\left|\left[\rho_{0}\right]\right| e^{-\gamma \rho_{-}^{\gamma-1} s}\left(\rho_{+}^{2(\gamma-1)}+\int_{0}^{1} u^{2} d x\right. \\
& \left.\quad+\rho_{-}^{-2} r_{-}^{-4} \int_{0}^{1} \rho^{2} u_{x}^{2} r^{4} d x\right) d s \\
& \leq \widehat{C}\left(1+\rho_{-}^{-2}\right)\left|\left[\rho_{0}\right]\right|\left(\rho_{-}^{1-\gamma} \rho_{+}^{2(\gamma-1)}+\rho_{-}^{1-\gamma}+\rho_{-}^{-2} r_{-}^{-4}\right) \leq \widehat{C},
\end{aligned}
$$

where $\widehat{C}$ is a positive constant independent of time and we assume that

$$
\begin{aligned}
0 & <\left|\left[\rho_{0}\right]\right| \\
& <\min \left\{\frac{\rho_{-}^{\gamma-1} \rho_{+}^{2(1-\gamma)}}{\left(1+\rho_{-}^{-2}\right)}, \frac{\rho_{-}^{\gamma-1}}{\left(1+\rho_{-}^{-2}\right)}, \frac{\rho_{-}^{2} r_{-}^{4}}{\left(1+\rho_{-}^{-2}\right)}\right\}:=M_{2} .
\end{aligned}
$$

The proof of (34) is completed.
Lemma 6. Let $T>0$. Under the conditions in Theorem 1, there exists a constant $\rho^{*}>0$ such that

$$
0<\rho(x, \tau) \leq \rho^{*}, \quad(x, \tau) \in[0,1] \times[0, T] .
$$

Proof. It follows from (19) and (34) that

$$
\begin{aligned}
\rho(x, \tau) & =\rho(r, t) \\
& \leq \int_{r_{-}}^{r_{+}} \rho(r, t) d r+\int_{r_{-}}^{r_{+}}\left|\rho_{r}(r, t)\right| d r \\
& \leq r_{-}^{-2} \int_{r_{-}}^{r_{+}} \rho(r, t) r^{2} d r+r_{-}^{-2} \int_{r_{-}}^{r_{+}} \rho^{1 / 2}\left|\left(\rho^{1 / 2}\right)_{r}(r, t)\right| r^{2} d r \\
& \leq C+C\left(f_{r_{-}}^{r_{+}} \rho r^{2} d r\right)^{1 / 2}\left(f_{r_{-}}^{r_{+}}\left|\left(\rho^{1 / 2}\right)_{r}(r, t)\right|^{2} r^{2} d r\right)^{1 / 2} \\
& \leq C+C \max \left(f_{0}^{1}\left|\rho_{x}(x, \tau)\right|^{2} r^{4} d x\right)^{1 / 2} \leq C,
\end{aligned}
$$

which yields

$$
\rho(x, \tau) \leq C:=\rho^{*} .
$$

Thus, we can choose $\rho_{+}=\rho^{*}+1$ to have

$$
0<\rho(x, \tau) \leq \rho^{*} \leq \rho_{+}, \quad(x, \tau) \in\left[0, x_{0}\right) \cap\left(x_{0}, 1\right] \times[0, T] .
$$

Lemma 7. Let $T>0$. Under the conditions in Theorem 1 , it holds for any solution $(\rho, u)$ to the initial boundary value problem (23) that

$$
\int_{0}^{1} u^{2 n} d x+\int_{0}^{\tau} f_{0}^{1}\left(\frac{u^{2 n}}{r^{2}}+\rho^{2} u^{2 n-2} u_{x}^{2} r^{4}\right) d x d s \leq C(T),
$$

for any positive integer $n \in N$, where $C(T)$ is a positive constant dependent of time.

Proof. Multiplying $(23)_{2}$ with $r^{2} u^{2 n-1}$, integrating by parts over $[0,1]$, we have

$$
\begin{aligned}
\frac{d}{d \tau} f_{0}^{1} & \frac{u^{2 n}}{2 n} d x+\int_{0}^{1} \rho^{2}\left(r^{2} u\right)_{x}\left(r^{2} u^{2 n-1}\right)_{x} d x \\
= & f_{0}^{1}\left(\rho^{\gamma}\left(r^{2} u^{2 n-1}\right)_{x}+\rho\left(2 r u^{2 n}\right)_{x}\right) d x \\
& +\left.2[\rho]\left(r u^{2 n}\right)\right|_{x=x_{0}} .
\end{aligned}
$$

Since it holds that

$$
\begin{aligned}
& \left(r^{2} u\right)_{x}\left(r^{2} u^{2 n-1}\right)_{x} \\
& =\left(\frac{2 u}{\rho r}+r^{2} u_{x}\right)\left(\frac{2 u^{2 n-1}}{\rho r}+(2 n-1) r^{2} u^{2 n-2} u_{x}\right) \\
& =\frac{4 u^{2 n}}{\rho^{2} r^{2}}+(2 n-1) u^{2 n-2} u_{x}^{2} r^{4}+\frac{4 n u^{2 n-1} u_{x} r}{\rho},
\end{aligned}
$$


it follows from (48) that

$$
\begin{aligned}
\frac{d}{d \tau} f_{0}^{1} & \frac{u^{2 n}}{2 n} d x+2 f_{0}^{1} \frac{u^{2 n}}{r^{2}} d x+(2 n-1) f_{0}^{1} \rho^{2} u^{2 n-2} u_{x}^{2} r^{4} d x \\
= & 2 f_{0}^{1} \frac{\rho^{\gamma-1} u^{2 n-1}}{r} d x+(2 n-1) f_{0}^{1} \rho^{\gamma} u^{2 n-2} u_{x} r^{2} d x \\
& +\left.2[\rho]\left(r u^{2 n}\right)\right|_{x=x_{0}} \\
\leq & \int_{0}^{1} \frac{u^{2 n}}{r^{2}} d x+\int_{0}^{1} \rho^{2} u^{2 n-2} u_{x}^{2} r^{4} d x+C\|\rho\|_{L^{\infty}}^{2(\gamma-1)} f_{0}^{1} u^{2 n-2} d x \\
& +\left.2[\rho]\left(r u^{2 n}\right)\right|_{x=x_{0}},
\end{aligned}
$$

which together with (43) and Young's inequality yields

$$
\begin{aligned}
& \frac{d}{d \tau} \int_{0}^{1} u^{2 n} d x+\int_{0}^{1} \frac{u^{2 n}}{r^{2}} d x+2(n-1) f_{0}^{1} u^{2 n-2} u_{x}^{2} r^{4} d x \\
& \quad \leq C+C \int_{0}^{1} u^{2 n} d x+\left.2[\rho]\left(r u^{2 n}\right)\right|_{x=x_{0}},
\end{aligned}
$$

and applying the Gronwall's inequality to (51), we can obtain

$$
\begin{aligned}
& \int_{0}^{1} u^{2 n} d x+\int_{0}^{\tau} f_{0}^{1} \frac{u^{2 n}}{r^{2}} d x d s \\
& +2(n-1) \int_{0}^{\tau} \int_{0}^{1} \rho^{2} u^{2 n-2} u_{x}^{2} r^{4} d x d s \\
& \quad \leq C(T)+\left.2 \int_{0}^{\tau}[\rho]\left(r u^{2 n}\right)\right|_{x=x_{0}} d s
\end{aligned}
$$

where $C(T)$ is a positive constant dependent of time. It holds from (24), (26), (30), and (31) that

$$
\begin{aligned}
& \left|\int_{0}^{\tau}[\rho]\left(r u^{2 n}\right)\right|_{x=x_{0}} d s \mid \\
& \leq\left.\widetilde{C}\left(1+\rho_{-}^{-1}\right)\right|_{0} ^{\tau}\left|\left[\rho_{0}\right]\right| e^{-\gamma \rho_{-}^{\gamma-1} s} \\
& \quad \times\left(\int_{0}^{1} u^{2 n} d x+f_{0}^{1} u^{2 n-2} u_{x}^{2} d x\right) d s \\
& \leq \widetilde{C}\left(1+\rho_{-}^{-1}\right)\left|\left[\rho_{0}\right]\right| \sup _{\tau \in[0, T]} \int_{0}^{1} u^{2 n} d x f_{0}^{\tau} e^{-\gamma \rho_{-}^{\gamma-1} s} d s \\
& \quad+\widetilde{C}\left(1+\rho_{-}^{-1}\right)\left|\left[\rho_{0}\right]\right| \rho_{-}^{-2} r_{-}^{-4} \int_{0}^{\tau} f_{0}^{1} \rho^{2} u^{2 n-2} u_{x}^{2} r^{4} d x d s \\
& \leq \frac{1}{2} \sup _{\tau \in[0, T]} f_{0}^{1} u^{2 n} d x+\frac{n-1}{2} \int_{0}^{\tau} f_{0}^{1} \rho^{2} u^{2 n-2} u_{x}^{2} r^{4} d x d s,
\end{aligned}
$$

where $\widetilde{C}$ is a positive constant independent of time and we assume that

$$
\begin{aligned}
0 & <\left|\left[\rho_{0}\right]\right| \\
& \leq \min \left\{\frac{\gamma}{2 \widetilde{C}\left(1+\rho_{-}^{-1}\right)} \rho_{-}^{\gamma-1}, \frac{(n-1)}{2 \widetilde{C}\left(1+\rho_{-}^{-1}\right)} \rho_{-}^{2} r_{-}^{4}\right\}:=M_{3} .
\end{aligned}
$$

From (52) and (53), we can deduce (47).

Lemma 8. Let $T>0$, for $n \in N$, and $n>1 / 2(\gamma-1)$. Under the conditions in Theorem 1 , it holds for any solution $(\rho, u)$ to the initial boundary value problem (23) that

$$
\begin{gathered}
\int_{0}^{\tau}\left\|\rho^{2 n(\gamma-1)} u^{2 n}\right\|_{L^{\infty}\left(\left[0, x_{0}\right) \cup\left(x_{0}, 1\right]\right)} d s \leq C(T), \\
\int_{0}^{\tau}\left\|\left|\left(\rho^{\gamma}\right)_{x} r^{2}\right|^{2 n}\right\|_{L^{\infty}\left(\left[0, x_{0}\right) \cup\left(x_{0}, 1\right]\right)} d s \leq C(T), \quad \tau \in[0, T],
\end{gathered}
$$

where $C(T)$ is a positive constant dependent of time.

Proof. By means of Sobolev imbedding theorem and CauchySchwartz inequality, applying (34), (43), and (47), we get

$$
\begin{aligned}
\int_{0}^{\tau} & \left\|\rho^{2 n(\gamma-1)} u^{2 n}\right\|_{L^{\infty}\left(\left[0, x_{0}\right) \cup\left(x_{0}, 1\right]\right)} d s \\
\leq & \int_{0}^{\tau} f_{0}^{1}\left|\rho^{2 n(\gamma-1)} u^{2 n}\right| d x d s+\int_{0}^{\tau} f_{0}^{1}\left|\left(\rho^{2 n(\gamma-1)} u^{2 n}\right)_{x}\right| d x d s \\
\leq & C(T)+C \int_{0}^{\tau} f_{0}^{1} \rho^{2 n(\gamma-1)-1}\left|\rho_{x}\right| u^{2 n} d x d s \\
& +C \int_{0}^{\tau} f_{0}^{1} \rho^{2 n(\gamma-1)}\left|u^{2 n-1} u_{x}\right| d x d s \\
\leq & C(T)+C \int_{0}^{\tau} f_{0}^{1} \rho_{x}^{2} r^{4} d x d s \\
& +C \int_{0}^{\tau} f_{0}^{1} \rho^{2(2 n(\gamma-1)-1)} u^{4 n} r^{-4} d x d s \\
& +\int_{0}^{\tau} f_{0}^{1} \rho^{2(2 n(\gamma-1)-1)} u^{2 n} r^{-4} d x d s \\
& +C \int_{0}^{\tau} f_{0}^{1} \rho^{2} u^{2 n-2} u_{x}^{2} r^{4} d x d s \leq C(T)
\end{aligned}
$$

Next, we find that

$$
\rho_{x} r^{2}=\rho_{0 x} r^{2}+u_{0}-u-\int_{0}^{\tau}\left(\rho^{\gamma}\right)_{x} r^{2} d s
$$


which together with (43), (47), and (57) gives

$$
\begin{aligned}
& \int_{0}^{\tau}\left\|\left|\left(\rho^{\gamma}\right)_{x} r^{2}\right|^{2 n}\right\|_{L^{\infty}} d s \\
& =\gamma^{2 n} \int_{0}^{\tau}\left\|\rho^{2 n(\gamma-1)}\left|\rho_{x} r^{2}\right|^{2 n}\right\|_{L^{\infty}} d s \\
& =\gamma^{2 n} \int_{0}^{\tau} \| \rho^{2 n(\gamma-1)} \mid \rho_{0 x} r^{2}+u_{0}-u \\
& \leq C \int_{0}^{\tau}\left\|\left.\rho^{2 n(\gamma-1)}\left(\rho_{0 x}^{2 n}+u_{0}^{2 n}+\rho^{\gamma}\right)_{x} r^{2} d l\right|^{2 n}\right\|_{L^{\infty}} d s \\
& +C \int_{0}^{\tau}\left\|\rho^{2 n(\gamma-1)}\left(\int_{0}^{s}\left(\rho^{\gamma}\right)_{x} r^{2} d l\right)^{2 n}\right\|_{L^{\infty}} d s \\
& \leq C(T)+C \int_{0}^{\tau}\left\|\rho^{2 n(\gamma-1)} u^{2 n}\right\|_{L^{\infty}} d s \\
& \quad+C(T) \int_{0}^{\tau} \int_{0}^{s}\left\|\left|\left(\rho^{\gamma}\right)_{x} r^{2}\right|^{2 n}\right\|_{L^{\infty}} d l d s,
\end{aligned}
$$

and applying the Gronwall's inequality to (59), we obtain (56).

Lemma 9. Let $T>0$. Under the conditions in Theorem 1, there exists a constant $\rho_{*}>0$ such that

$$
\rho(x, \tau) \geq \rho_{*}>0, \quad(x, \tau) \in[0,1] \times[0, T] .
$$

Proof. It is easy to verify

$$
\begin{aligned}
& \overline{\rho_{L}^{(\gamma+1) / 2}}(\tau):=\frac{1}{x_{0}} \int_{0}^{x_{0}} \rho^{(\gamma+1) / 2}(x, \tau) d x \\
& \geq \frac{r_{-}^{2}}{r_{+}^{2} x_{0}}\left(\int_{r_{-}}^{\xi(t)} \rho(r, t) r^{2} d r\right)^{(\gamma+3) / 2} \\
&=\frac{r_{-}^{2}}{r_{+}^{2} x_{0}}\left(\int_{r_{-}}^{\xi_{0}} \rho_{0}(r) r^{2} d r\right)^{(\gamma+3) / 2}>0, \\
&\left\|\rho^{(\gamma+1) / 2}-\overline{\rho_{L}^{(\gamma+1) / 2}}(\tau)\right\|_{L^{2}\left(\left[0, x_{0}\right)\right)}^{2} \in W^{1,1}([0, T]) .
\end{aligned}
$$

Indeed, it holds that

$$
\begin{aligned}
& \int_{0}^{\tau}\left\|\rho^{(\gamma+1) / 2}-\overline{\rho_{L}^{(\gamma+1) / 2}}(s)\right\|_{L^{2}\left(\left[0, x_{0}\right)\right)}^{2} d s \\
& \quad \leq C \int_{0}^{\tau}\left\|\left(\rho^{(\gamma+1) / 2}\right)_{x} r^{2}\right\|_{L^{2}\left(\left[0, x_{0}\right)\right)}^{2} d s \leq C,
\end{aligned}
$$

which together with (23), (34), and (43) gives

$$
\begin{gathered}
\int_{0}^{\tau}\left|\frac{d}{d s}\left\|\rho^{(\gamma+1) / 2}-\overline{\rho_{L}^{(\gamma+1) / 2}}(s)\right\|_{L^{2}\left(\left[0, x_{0}\right)\right)}^{2}\right| d s \\
\leq(\gamma+1) \int_{0}^{\tau} \mid \int_{0}^{x_{0}}\left(\rho^{(\gamma+1) / 2}-\overline{\rho_{L}^{(\gamma+1) / 2}}(s)\right) \\
\times \rho^{(\gamma-1) / 2} \rho_{s} d x \mid d s \\
+2 \int_{0}^{\tau} \mid \int_{0}^{x_{0}}\left(\rho^{(\gamma+1) / 2}-\overline{\rho_{L}^{(\gamma+1) / 2}}(s)\right) \\
\leq C \int_{0}^{\tau} \int_{0}^{x_{0}}\left(\rho^{(\gamma+1) / 2}-\overline{\rho_{L}^{(\gamma+1) / 2}}(s)\right)^{2} d x d s \\
+C \int_{0}^{\tau} f_{0}^{x_{0}}\left(\frac{u^{2}}{r^{2}}+\rho^{2} u_{x}^{2} r^{4}\right) d x d s \leq C .
\end{gathered}
$$

Similarly, we can obtain

$$
\left\|\rho^{(\gamma+1) / 2}-\overline{\rho_{R}^{(\gamma+1) / 2}}(\tau)\right\|_{L^{2}\left(\left(x_{0}, 1\right]\right)}^{2} \in W^{1,1}([0, T]),
$$

where we have used

$$
\begin{aligned}
\overline{\rho_{R}^{(\gamma+1) / 2}}(\tau) & :=\frac{1}{1-x_{0}} \int_{x_{0}}^{1} \rho^{(\gamma+1) / 2}(x, \tau) d x \\
& \geq \frac{r_{-}^{2}}{r_{+}^{2}\left(1-x_{0}\right)}\left(\int_{\xi(t)}^{r_{+}} \rho(r, t) r^{2} d r\right)^{(\gamma+3) / 2} \\
& =\frac{r_{-}^{2}}{r_{+}^{2} x_{0}}\left(\int_{\xi_{0}}^{r_{+}} \rho_{0}(r) r^{2} d r\right)^{(\gamma+3) / 2}>0 .
\end{aligned}
$$

By Gagliardo-Nirenberg-Sobolev inequality, (62), and (65), we have

$$
\begin{aligned}
& \left\|\rho^{(\gamma+1) / 2}-\overline{\rho_{L}^{(\gamma+1) / 2}}(\tau)\right\|_{L^{\infty}\left(\left[0, x_{0}\right)\right)}^{2} \\
& \quad+\left\|\rho^{(\gamma+1) / 2}-\overline{\rho_{R}^{(\gamma+1) / 2}}(\tau)\right\|_{L^{\infty}\left(\left(x_{0}, 1\right]\right)}^{2} \longrightarrow 0 \quad \text { as } \tau \longrightarrow+\infty .
\end{aligned}
$$

Thus, there is a $T_{0}>0$ and a constant $\rho_{1}>0$ such that

$$
\rho(x, \tau) \geq \rho_{1}, \quad x \in\left[0, x_{0}\right) \cup\left(x_{0}, 1\right], \tau \in\left[T_{0},+\infty\right) .
$$

For $\tau \in\left[0, T_{0}\right]$, denote

$$
v(x, \tau)=\frac{1}{\rho(x, \tau)},
$$

and then from (23), we can obtain

$$
v_{t}=\left(r^{2} u\right)_{x}
$$


and multiplying (70) by $4 v^{3}$ and integrating the result over $\left(\left[0, x_{0}\right) \cup\left(x_{0}, 1\right]\right) \times\left[0, T_{0}\right]$, we have

$$
\begin{aligned}
f_{0}^{1} v^{4} d x= & f_{0}^{1} v_{0}^{4} d x+4 \int_{0}^{\tau} f_{0}^{1} v^{3}\left(r^{2} u\right)_{x} d x d s \\
= & f_{0}^{1} v_{0}^{4} d x-\left.4 \int_{0}^{\tau}\left[v^{3}\right]\left(r^{2} u\right)\right|_{x=x_{0}} d s \\
& +12 \int_{0}^{\tau} f_{0}^{1} v^{4} u \rho_{x} r^{2} d x d s \\
= & \int_{0}^{1} v_{0}^{4} d x-\left.4 \int_{0}^{\tau}\left[v^{3}\right]\left(r^{2} u\right)\right|_{x=x_{0}} d s \\
& +12 \int_{0}^{\tau} f_{0}^{1} v^{4} u\left(\rho_{0 x} r^{2}+u_{0}-u\right. \\
& \left.-\int_{0}^{s}\left(\rho^{\gamma}\right)_{x} r^{2} d l\right) d x d s .
\end{aligned}
$$

From (56), it holds that

$$
\begin{aligned}
f_{0}^{1} v^{4} d x & +12 \int_{0}^{\tau} f_{0}^{1} v^{4} u^{2} d x d s \\
= & f_{0}^{1} v_{0}^{4} d x-\left.4 \int_{0}^{\tau}\left[v^{3}\right]\left(r^{2} u\right)\right|_{x=x_{0}} d s \\
& +12 \int_{0}^{\tau} f_{0}^{1} v^{4} u \rho_{0 x} r^{2} d x d s \\
& +12 \int_{0}^{\tau} f_{0}^{1} v^{4} u u_{0} d x d s \\
& -12 \int_{0}^{\tau} f_{0}^{1} v^{4} u\left(\int_{0}^{s}\left(\rho^{\gamma}\right)_{x} r^{2} d l\right) d x d s \\
\leq & 6 \int_{0}^{\tau} f_{0}^{1} v^{4} u^{2} d x d s+C\left(T_{0}\right) \int_{0}^{\tau} f_{0}^{1} v^{4} d x d s \\
& +C\left|\int_{0}^{\tau}\left[v^{3}\right]\left(r^{2} u\right)\right|_{x=x_{0}} d s \mid .
\end{aligned}
$$

It holds from (24), (26), (30), and (31) that

$$
\begin{aligned}
& \left|\int_{0}^{\tau}\left[v^{3}\right]\left(r^{2} u\right)\right|_{x=x_{0}} d s \mid \\
& \leq \check{C}\left(1+\rho_{-}^{-2}\right) \int_{0}^{\tau} \rho_{+}^{-4}|[\rho]|\left(f_{0}^{1} u^{2} d x+\int_{0}^{1} u_{x}^{2} d x\right)^{1 / 2} d s \\
& \quad \leq \check{C}\left(1+\rho_{-}^{-2}\right) \int_{0}^{\tau}\left|\left[\rho_{0}\right]\right| e^{-\gamma \rho_{-}^{\gamma-1} s}\left(\rho_{+}^{-8}+f_{0}^{1} u^{2} d x\right. \\
& \left.\quad+\rho_{-}^{-2} r_{-}^{-4} f_{0}^{1} \rho^{2} u_{x}^{2} r^{4} d x\right) d s \\
& \leq \check{C}\left(1+\rho_{-}^{-2}\right)\left|\left[\rho_{0}\right]\right|\left(\rho_{-}^{1-\gamma} \rho_{+}^{-8}+\rho_{-}^{1-\gamma}+\rho_{-}^{-2} r_{-}^{-4}\right) \leq \check{C},
\end{aligned}
$$

where $\check{C}$ is a positive constant independent of time and we assume that

$$
\begin{aligned}
0 & <\left|\left[\rho_{0}\right]\right| \\
& <\min \left\{M_{1}, M_{2}, M_{3}, \frac{\rho_{-}^{\gamma-1} \rho_{+}^{8}}{\left(1+\rho_{-}^{-2}\right)}, \frac{\rho_{-}^{\gamma-1}}{\left(1+\rho_{-}^{-2}\right)}, \frac{\rho_{-}^{2} r_{-}^{4}}{\left(1+\rho_{-}^{-2}\right)}\right\} \\
& :=\delta .
\end{aligned}
$$

Then, it follows from (72) and (73) that

$$
\int_{0}^{1} v^{4} d x \leq C+C\left(T_{0}\right) \int_{0}^{\tau} f_{0}^{1} v^{4} d x d s
$$

where $C\left(T_{0}\right)$ is a positive constant independent of time $T_{0}$. By Gronwall's inequality, (75) leads to

$$
f_{0}^{1} v^{4} d x=f_{0}^{1} \frac{1}{\rho^{4}} d x \leq C\left(T_{0}\right) .
$$

It holds for $(x, \tau) \in\left(\left[0, x_{0}\right) \cup\left(x_{0}, 1\right]\right) \times\left[0, T_{0}\right]$ that

$$
\begin{aligned}
\frac{1}{\rho} & =f_{0}^{1} \frac{1}{\rho} d x+\int_{0}^{1}\left|\left(\frac{1}{\rho}\right)_{x}\right| d x \\
& \leq C+C\left(f_{0}^{1} \frac{1}{\rho^{4}} d x\right)^{1 / 2}\left(f_{0}^{1} \rho_{x}^{2} r^{4} d x\right)^{1 / 2} \leq C\left(T_{0}\right) ;
\end{aligned}
$$

namely,

$$
\begin{aligned}
& \rho(x, \tau) \geq C\left(T_{0}\right):=\rho_{2}, \\
& (x, \tau) \in\left(\left[0, x_{0}\right) \cup\left(x_{0}, 1\right]\right) \times\left[0, T_{0}\right] .
\end{aligned}
$$

Therefore, we can choose

$$
\rho_{*}=\min \left\{\rho_{1}, \rho_{2}\right\}, \quad \rho_{-}=\frac{1}{2} \rho_{*},
$$

to get

$$
\rho \geq \rho_{*}>\rho_{-}, \quad(x, \tau) \in\left(\left[0, x_{0}\right) \cup\left(x_{0}, 1\right]\right) \times[0,+\infty) .
$$

Lemma 10. Let $T>0$. Under the conditions in Theorem 1, it holds for any solution $(\rho, u)$ to the initial boundary value problem (23) that

$$
\begin{array}{r}
\left|\left[\rho_{0}\left(x_{0}\right)\right]\right| e^{-C_{0} \tau} \leq\left|\left[\rho\left(x_{0}, \tau\right)\right]\right| \leq\left|\left[\rho_{0}\left(x_{0}\right)\right]\right| e^{-C_{1} \tau}, \\
\tau \in[0, T],
\end{array}
$$

where $C_{0}, C_{1}$ are positive constants independent of time.

Proof. From (30), (31), (43), and (60), we can get (81). 
Abstract and Applied Analysis

9

Lemma 11. Let $T>0$. Under the conditions in Theorem 1, it holds for any solution $(\rho, u)$ to the initial boundary value problem (23) that

$$
\begin{array}{r}
f_{0}^{1}\left(r^{2} u\right)_{x}^{2} d x+f_{0}^{1}\left(r^{2} u\right)_{\tau}^{2} r^{-4} d x+\int_{0}^{\tau} f_{0}^{1}\left(r^{2} u\right)_{s}^{2} r^{-4} d x d s \\
+\int_{0}^{\tau} f_{0}^{1} \rho^{2}\left(r^{2} u\right)_{x s}^{2} d x d s+\int_{0}^{\tau} f_{0}^{1}\left(r^{2} u\right)_{x x}^{2} d x d s \leq C, \\
\tau \in[0, T],
\end{array}
$$

where $C>0$ denotes a constant independent of time.

Proof. Multiplying $(23)_{2}$ by $\rho^{-2}\left(r^{2} u\right)_{\tau}$ and integrating the result with respect to $x$ over $[0,1]$, making use of (21) and (24), it holds that

$$
\begin{aligned}
\frac{d}{d \tau} f_{0}^{1}( & \left.\frac{1}{2}\left(r^{2} u\right)_{x}^{2}-\rho^{\gamma-2}\left(r^{2} u\right)_{x}\right) d x+f_{0}^{1} \rho^{-2}\left(r^{2} u\right)_{\tau}^{2} r^{-4} d x \\
= & (\gamma-2) f_{0}^{1} \rho^{\gamma-1}\left(r^{2} u\right)_{x}^{2} d x-2 f_{0}^{1} \rho^{\gamma-3} \rho_{x}\left(r^{2} u\right)_{\tau} d x \\
& +2 f_{0}^{1} \rho^{-1} \rho_{x}\left(r^{2} u\right)_{x}\left(r^{2} u\right)_{\tau} d x \\
& +2 f_{0}^{1} \rho^{-2} u^{2}\left(r^{2} u\right)_{\tau} r^{-3} d x-2 f_{0}^{1} \rho^{-2} \rho_{x} u\left(r^{2} u\right)_{\tau} r^{-1} d x \\
& +\left.\left[\rho^{-2}\right]\left(\left(\rho^{\gamma}-\rho^{2}\left(r^{2} u\right)_{x}\right)\left(r^{2} u\right)_{\tau}\right)\right|_{x=x_{0}^{+}}
\end{aligned}
$$

which gives

$$
\begin{aligned}
f_{0}^{1}\left(r^{2} u\right)_{x}^{2} d x+\int_{0}^{\tau} f_{0}^{1}\left(r^{2} u\right)_{s}^{2} r^{-4} d x d s \\
\leq C+C f_{0}^{1} \rho^{2(\gamma-2)} d x+C \int_{0}^{\tau} f_{0}^{1}\left(\frac{u^{2}}{r^{2}}+u_{x}^{2} r^{4}\right) d x d s \\
+C \int_{0}^{\tau} f_{0}^{1} \rho_{x}^{2} r^{4} d x d s+C \int_{0}^{\tau} f_{0}^{1} \rho_{x}^{2}\left(r^{2} u\right)_{x}^{2} r^{4} d x d s \\
+C \int_{0}^{\tau} f_{0}^{1} u^{4} r^{-2} d x d s+C \int_{0}^{\tau} f_{0}^{1} \rho_{x}^{2} u^{2} r^{2} d x d s \\
+\int_{0}^{\tau}\left|\left[\rho^{-2}\right]\left(\left(\rho^{\gamma}-\rho^{2}\left(r^{2} u\right)_{x}\right)\left(r^{2} u\right)_{s}\right)\right|_{x=x_{0}^{+}} \mid d s \\
\leq C+C \int_{0}^{\tau} f_{0}^{1} \rho_{x}^{2}\left(r^{2} u\right)_{x}^{2} r^{4} d x d s+C \sup _{\tau \in[0, T]}\|u\|_{L^{\infty}}^{2} \\
+\int_{0}^{\tau}\left|\left[\rho^{-2}\right]\left(\left(\rho^{\gamma}-\rho^{2}\left(r^{2} u\right)_{x}\right)\left(r^{2} u\right)_{s}\right)\right|_{x=x_{0}^{+}} \mid d s .
\end{aligned}
$$

It holds from $(23)_{2},(26),(34)$, and (43) that

$$
\begin{aligned}
& C \int_{0}^{\tau} f_{0}^{1} \rho_{x}^{2}\left(r^{2} u\right)_{x}^{2} r^{4} d x d s \\
& \leq \frac{C}{3} \int_{0}^{\tau} f_{0}^{1} \rho_{x}^{2}\left(r^{2} u\right)_{x}^{2} r^{4} d x d s+\frac{1}{6} \int_{0}^{\tau} f_{0}^{1}\left(r^{2} u\right)_{s}^{2} r^{-4} d x d s \\
& +C \int_{0}^{\tau} f_{0}^{1} \rho_{x}^{2} d x d s+C \int_{0}^{\tau} f_{0}^{1}\left(\frac{u^{2}}{r^{2}}+u_{x}^{2} r^{4}\right) d x d s, \\
& C \sup _{\tau \in[0, T]}\|u\|_{L^{\infty}}^{2} \leq \frac{1}{4} \sup _{\tau \in[0, T]} f_{0}^{1}\left(r^{2} u\right)_{x}^{2} d x+C \sup _{\tau \in[0, T]} f_{0}^{1} u^{2} d x, \\
& \int_{0}^{\tau}\left|\left[\rho \rho^{-2}\right]\left(\left(\rho^{\gamma}-\rho^{2}\left(r^{2} u\right)_{x}\right)\left(r^{2} u\right)_{s}\right)\right|_{x=x_{0}^{+}} \mid d s \\
& \leq C \int_{0}^{\tau}\left\|\left[\rho_{0}\right] \mid e^{-C s}\right\| \rho^{\gamma}-\rho^{2}\left(r^{2} u\right)_{x}\left\|_{L^{\infty}}\right\|\left(r^{2} u\right)_{s} \|_{L^{\infty}} d s \\
& \leq C \int_{0}^{\tau} e^{-C s}\left\|\rho^{\gamma}\right\|_{L^{\infty}}^{2} d s+C \int_{0}^{\tau} f_{0}^{1} \rho_{x}^{2} d x d s \\
& \quad+C \int_{0}^{\tau} f_{0}^{1}\left(\frac{u^{2}}{r^{2}}+u_{x}^{2} r^{4}\right) d x d s \\
& \quad \frac{1}{4} \int_{0}^{\tau} f_{0}^{1}\left(r^{2} u\right)_{s}^{2} r^{-4} d x d s+C \varepsilon \int_{0}^{\tau} f_{0}^{1}\left(r^{2} u\right)_{x s}^{2} r^{-4} d x d s, \\
& \quad+87)
\end{aligned}
$$

where $C$ denotes a positive constant independent of time and $\varepsilon \in(0,1)$ is a small constant which will be chosen later. It holds from (84)-(87) that

$$
\begin{gathered}
f_{0}^{1}\left(r^{2} u\right)_{x}^{2} d x+\int_{0}^{\tau} f_{0}^{1}\left(r^{2} u\right)_{s}^{2} r^{-4} d x d s \\
\leq C+C \varepsilon \int_{0}^{\tau} f_{0}^{1}\left(r^{2} u\right)_{x s}^{2} r^{-4} d x d s .
\end{gathered}
$$

Differentiating $(23)_{2}$ with respect to $\tau$, multiplying the result by $\left(r^{2} u\right)_{\tau}$, and integrating the result with respect to $x$ over $[0,1]$, we deduce

$$
\begin{aligned}
& \frac{1}{2} \frac{d}{d \tau} f_{0}^{1}\left(r^{2} u\right)_{\tau}^{2} r^{-4} d x+f_{0}^{1} \rho^{2}\left(r^{2} u\right)_{x \tau}^{2} d x \\
& =2 f_{0}^{1} u u_{\tau}\left(r^{2} u\right)_{\tau} r^{-3} d x-\frac{1}{2} f_{0}^{1}\left(r^{-4}\right)_{\tau}\left(r^{2} u\right)_{\tau}^{2} d x \\
& \quad+2 f_{0}^{1} u\left(r^{-1} u\right)_{\tau}\left(r^{2} u\right)_{\tau} r^{-2} d x \\
& \quad+f_{0}^{1}\left(\rho^{\gamma}\right)_{\tau}\left(r^{2} u\right)_{x \tau} d x-f_{0}^{1}\left(\rho^{2}\right)_{\tau}\left(r^{2} u\right)_{x}\left(r^{2} u\right)_{x \tau} d x \\
& \quad-f_{0}^{1}\left(\frac{2 \rho_{x} u}{r}\right)_{\tau}\left(r^{2} u\right)_{\tau} d x+2 f_{0}^{1}\left(r^{-2}\right)_{\tau} r^{-1} u^{2}\left(r^{2} u\right)_{\tau} d x .
\end{aligned}
$$


A complicated computation implies

$$
\begin{aligned}
& \frac{d}{d \tau} f_{0}^{1}\left(r^{2} u\right)_{\tau}^{2} r^{-4} d x+f_{0}^{1} \rho^{2}\left(r^{2} u\right)_{x \tau}^{2} d x \\
& \leq C f_{0}^{1}\left(r^{2} u\right)_{\tau}^{2} r^{-4} d x \\
& \quad+C f_{0}^{1}\left(\frac{u^{2}}{r^{2}}+u_{x}^{2} r^{4}\right) d x f_{0}^{1}\left(r^{2} u\right)_{\tau}^{2} r^{-4} d x \\
& +C \sup _{\tau \in[0, T]}\left\|\left(r^{2} u\right)_{x}^{2}\right\|_{L^{\infty}} f_{0}^{1}\left(r^{2} u\right)_{x}^{2} d x \\
& +C f_{0}^{1}\left(\frac{u^{2}}{r^{2}}+u_{x}^{2} r^{4}\right) d x,
\end{aligned}
$$

by means of Gronwall's inequality, $(23)_{2},(26),(34),(43)$, and (88), we have

$$
\begin{aligned}
& f_{0}^{1}\left(r^{2} u\right)_{\tau}^{2} r^{-4} d x+\int_{0}^{\tau} f_{0}^{1} \rho^{2}\left(r^{2} u\right)_{x s}^{2} d x d s \\
& \leq C+C \varepsilon \int_{0}^{\tau} f_{0}^{1} \rho^{2}\left(r^{2} u\right)_{x s}^{2} d x d s+C \sup _{\tau \in[0, T]}\left\|\left(r^{2} u\right)_{x}^{2}\right\|_{L^{\infty}} \\
& \leq C+C \varepsilon \int_{0}^{\tau} f_{0}^{1} \rho^{2}\left(r^{2} u\right)_{x s}^{2} d x d s \\
& +C \sup _{\tau \in[0, T]}\left(f_{0}^{1}\left(r^{2} u\right)_{x}^{2} d x\right)^{1 / 2}\left(f_{0}^{1}\left(r^{2} u\right)_{x x}^{2} d x\right)^{1 / 2} \\
& \leq C+C \varepsilon \int_{0}^{\tau} f_{0}^{1} \rho^{2}\left(r^{2} u\right)_{x s}^{2} d x d s+\frac{1}{2} f_{0}^{1}\left(r^{2} u\right)_{\tau}^{2} r^{-4} d x
\end{aligned}
$$

where $C$ denotes a positive constant independent of time; choosing the constant $\varepsilon$ small sufficiently, we complete the proof of Lemma 11.

Remark 12. By Lemmas 4-11, the following inequality holds:

$$
\begin{gathered}
f_{0}^{1} u^{2} d x+f_{0}^{1} u_{x}^{2} d x+f_{0}^{1} u_{\tau}^{2} d x+f_{0}^{1} \rho_{x}^{2} d x+\int_{0}^{\tau} f_{0}^{1} \rho_{x}^{2} d x d s \\
\quad+\int_{0}^{\tau} f_{0}^{1} u_{x}^{2} d x d s+\int_{0}^{\tau} f_{0}^{1} u_{s}^{2} d x d s \\
\quad+\int_{0}^{\tau} f_{0}^{1} u_{x x}^{2} d x d s+\int_{0}^{\tau} f_{0}^{1} u_{x \tau}^{2} d x d s \leq C .
\end{gathered}
$$

Remark 13. In fact, there are several fundamental estimates, such as (26) in Lemma 4, (34) in Lemma 5, (43) in Lemma 6, (47) in Lemma 7, (60) in Lemma 9, and (82) in Lemma 11, which can be also found in $[15,20,22,26]$ and so forth.

Lemma 14. Under the conditions in Theorem 1, it holds for any solution $(\rho, u)$ to the initial boundary value problem (7)-(8) that

$$
\|(\rho-\bar{\rho}, u)(\cdot, t)\|_{L^{\infty}\left(\left[r_{-}, \xi(t)\right) \cup\left(\xi(t), r_{+}\right]\right)} \leq C_{2} e^{-C_{3} t}, \quad t>0,
$$

where $C_{2}$ and $C_{3}$ denote the constants independent of time and $\bar{\rho}=1 /\left(r_{+}-r_{-}\right) f_{r_{-}}^{r_{+}} \rho(r, t) r^{2} d r$.

Proof. In a similar argument to show (28) and (40) with modification, we can obtain (94) and (95)

$$
\begin{aligned}
& \frac{d}{d t} f_{r_{-}}^{r_{+}}\left(\frac{1}{2} \rho u^{2}+\frac{1}{\gamma-1}\left(\rho^{\gamma-1}-\bar{\rho}^{\gamma-1}-\gamma \bar{\rho}^{\gamma-1}(\rho-\bar{\rho})\right)\right) r^{2} d r \\
& \quad+\int_{r_{-}}^{r_{+}}\left(2 \rho u^{2}+\rho u_{r}^{2} r^{2}\right) d r=\left.2 \xi(t)[\rho] u^{2}\right|_{r=\xi(t)}, \\
& \frac{1}{2} \frac{d}{d t} \int_{r_{-}}^{r_{+}} \rho\left(u+\rho^{-1} \rho_{r}\right)^{2} r^{2} d r \\
& \quad+\frac{1}{\gamma-1} \frac{d}{d t} \int_{r_{-}}^{r_{+}}\left(\rho^{\gamma-1}-\bar{\rho}^{\gamma-1}-\gamma \bar{\rho}^{\gamma-1}(\rho-\bar{\rho})\right) r^{2} d r \\
& \quad+\gamma f_{r_{-}}^{r_{+}} \rho^{\gamma-2} \rho_{r}^{2} r^{2} d r=\left.\xi(t)^{2}\left[\rho^{\gamma}\right] u\right|_{r=\xi(t)} d s .
\end{aligned}
$$

It holds from (34), (43), and (60) that

$$
f_{r_{-}}^{r_{+}}(\rho-\bar{\rho})^{2} r^{2} d r \leq C \int_{r_{-}}^{r_{+}} \rho^{\gamma-2} \rho_{r}^{2} r^{2} d r .
$$

Denote

$$
\begin{aligned}
E(t):= & f_{r_{-}}^{r_{+}}\left(\rho u^{2}+\left(u+\rho^{-1} \rho_{r}\right)^{2}\right) r^{2} d r \\
& +f_{r_{-}}^{r_{+}}\left(\rho^{\gamma-1}-\bar{\rho}^{\gamma-1}-\gamma \bar{\rho}^{\gamma-1}(\rho-\bar{\rho})\right) r^{2} d r .
\end{aligned}
$$

By (94)-(97), a complicated computation gives rise to

$$
\frac{d}{d t} E(t)+\widetilde{C} E(t) \leq C\left(\left|[\rho] u^{2}\right|+\left|\left[\rho^{\gamma}\right] u\right|\right)
$$

where $\widetilde{C}<C_{1}$ is a positive constant and $C_{1}$ is the constant obtained in Lemma 10. From (97), we have

$$
\begin{aligned}
E(t) & \leq E(0) e^{-\widetilde{C} t}+C e^{-\widetilde{C} t} \int_{0}^{t} e^{\widetilde{C} s}\left(\left|[\rho] u^{2}\right|+\left|\left[\rho^{\gamma}\right] u\right|\right) d s \\
& \leq C_{2} e^{-C_{3} t}
\end{aligned}
$$

where we have used (26), (30), and (31). By the fact

$$
\begin{aligned}
E(t) \geq c( & \|u\|_{L^{2}\left(\left[r_{-}, \xi(t)\right) \cup\left(\xi(t), r_{+}\right]\right)}^{2}+\|\rho-\bar{\rho}\|_{L^{2}\left(\left[r_{-}, \xi(t)\right) \cup\left(\xi(t), r_{+}\right]\right)}^{2} \\
& \left.+\left\|\rho_{r}\right\|_{L^{2}\left(\left[r_{-}, \xi(t)\right) \cup\left(\xi(t), r_{+}\right]\right)}^{2}\right),
\end{aligned}
$$


where $c>0$ is a constant independent of time and GagliardoNirenberg-Sobolev inequality

$$
\begin{aligned}
& \|(\rho-\bar{\rho}, u)\|_{L^{\infty}\left(\left[r_{-}, \xi(t)\right) \cup\left(\xi(t), r_{+}\right]\right)} \\
& \leq C\|(\rho-\bar{\rho}, u)\|_{L^{2}\left(\left[r_{-}, \xi(t)\right) \cup\left(\xi(t), r_{+}\right]\right)}^{1 / 2} \\
& \quad \times\left\|(\rho-\bar{\rho}, u)_{r}\right\|_{L^{2}\left(\left[r_{-}, \xi(t)\right) \cup\left(\xi(t), r_{+}\right]\right)}^{1 / 2}
\end{aligned}
$$

we obtain (93).

\section{Proof of the Main Results}

Proof. The global existence of unique piecewise regular solution to the initial boundary value problem (7)-(8) can be established in terms of the short time existence carried out as in $[2,10]$, the uniform a priori estimates, and the analysis of regularities, which indeed follow from Lemmas $4-11$. In addition, one can show that $(\rho, u)$ is the global weak solution to the initial boundary value problem (7)-(8) with the initial data satisfying (9). We omit the details. The large time behaviors follow from Lemma 14 directly. The proof of Theorem 1 is completed.

\section{Conflict of Interests}

The authors declare that there is no conflict of interests regarding the publication of this paper.

\section{Acknowledgments}

The research of R. X. Lian is supported by NNSFC no. 11101145, China Postdoctoral Science Foundation no. 2012M520360, Doctoral Foundation of North China University of Water Sources and Electric Power no. 201032, and Innovation Scientists and Technicians Troop Construction Projects of Henan Province.

\section{References}

[1] D. Hoff, "Construction of solutions for compressible, isentropic Navier-Stokes equations in one space dimension with nonsmooth initial data," Proceedings of the Royal Society of Edinburgh A: Mathematics, vol. 103, no. 3-4, pp. 301-315, 1986.

[2] D. Hoff, "Global existence for 1D, compressible, isentropic Navier-Stokes equations with large initial data," Transactions of the American Mathematical Society, vol. 303, no. 1, pp. 169-181, 1987.

[3] D. Hoff, "Global well-posedness of the Cauchy problem for the Navier-Stokes equations of nonisentropic flow with discontinuous initial data," Journal of Differential Equations, vol. 95, no. 1, pp. 33-74, 1992.

[4] D. Hoff, "Spherically symmetric solutions of the Navier-Stokes equations for compressible, isothermal flow with large, discontinuous initial data," Indiana University Mathematics Journal, vol. 41, no. 4, pp. 1225-1302, 1992.

[5] D. Hoff, "Global solutions of the Navier-Stokes equations for multidimensional compressible flow with discontinuous initial data," Journal of Differential Equations, vol. 120, no. 1, pp. 215$254,1995$.
[6] D. Hoff, "Strong convergence to global solutions for multidimensional flows of compressible, viscous fluids with polytropic equations of state and discontinuous initial data," Archive for Rational Mechanics and Analysis, vol. 132, no. 1, pp. 1-14, 1995.

[7] G.-Q. Chen, D. Hoff, and K. Trivisa, "Global solutions of the compressible Navier-Stokes equations with large discontinuous initial data," Communications in Partial Differential Equations, vol. 25, no. 11-12, pp. 2233-2257, 2000.

[8] D. Hoff, "Discontinuous solutions of the Navier-Stokes equations for multidimensional flows of heat-conducting fluids," Archive for Rational Mechanics and Analysis, vol. 139, no. 4, pp. 303-354, 1997.

[9] D. Hoff, "Dynamics of singularity surfaces for compressible, viscous flows in two space dimensions," Communications on Pure and Applied Mathematics, vol. 55, no. 11, pp. 1365-1407, 2002.

[10] D. Fang and T. Zhang, "Global solutions of the Navier-Stokes equations for compressible flow with density-dependent viscosity and discontinuous initial data," Journal of Differential Equations, vol. 222, no. 1, pp. 63-94, 2006.

[11] R. Lian, Z. Guo, and H.-L. Li, "Dynamical behaviors for 1D compressible Navier-Stokes equations with density-dependent viscosity," Journal of Differential Equations, vol. 248, no. 8, pp. 1926-1954, 2010

[12] J.-F. Gerbeau and B. Perthame, "Derivation of viscous SaintVenant system for laminar shallow water; numerical validation," Discrete and Continuous Dynamical Systems B, vol. 1, no. 1, pp. 89-102, 2001.

[13] F. Marche, "Derivation of a new two-dimensional viscous shallow water model with varying topography, bottom friction and capillary effects," European Journal of Mechanics B: Fluids, vol. 26, no. 1, pp. 49-63, 2007.

[14] Z. Guo, Q. Jiu, and Z. Xin, "Spherically symmetric isentropic compressible flows with density-dependent viscosity coefficients," SIAM Journal on Mathematical Analysis, vol. 39, no. 5, pp. 1402-1427, 2008.

[15] Z. Guo, H.-L. Li, and Z. Xin, "Lagrange structure and dynamics for solutions to the spherically symmetric compressible NavierStokes equations," Communications in Mathematical Physics, vol. 309, no. 2, pp. 371-412, 2012.

[16] S. Jiang, Z. Xin, and P. Zhang, "Global weak solutions to 1D compressible isentropic Navier-Stokes equations with densitydependent viscosity," Methods and Applications of Analysis, vol. 12, no. 3, pp. 239-252, 2005.

[17] T.-P. Liu, Z. Xin, and T. Yang, "Vacuum states for compressible flow," Discrete and Continuous Dynamical Systems, vol. 4, no. 1, pp. 1-32, 1998.

[18] M. Okada, Š. M. Nečasová, and T. Makino, "Free boundary problem for the equation of one-dimensional motion of compressible gas with density-dependent viscosity," Annali dell'Università di Ferrara: Nuova Serie: Sezione VII. Scienze Matematiche, vol. 48, pp. 1-20, 2002.

[19] S.-W. Vong, T. Yang, and C. Zhu, "Compressible Navier-Stokes equations with degenerate viscosity coefficient and vacuum. II," Journal of Differential Equations, vol. 192, no. 2, pp. 475-501, 2003.

[20] T. Yang, Z.-a. Yao, and C. Zhu, "Compressible Navier-Stokes equations with density-dependent viscosity and vacuum," Communications in Partial Differential Equations, vol. 26, no. 5-6, pp. 965-981, 2001. 
[21] T. Yang and H. Zhao, "A vacuum problem for the one-dimensional compressible Navier-Stokes equations with densitydependent viscosity," Journal of Differential Equations, vol. 184, no. 1, pp. 163-184, 2002.

[22] T. Yang and C. Zhu, "Compressible Navier-Stokes equations with degenerate viscosity coefficient and vacuum," Communications in Mathematical Physics, vol. 230, no. 2, pp. 329-363, 2002.

[23] A. Mellet and A. Vasseur, "Existence and uniqueness of global strong solutions for one-dimensional compressible NavierStokes equations," SIAM Journal on Mathematical Analysis, vol. 39, no. 4, pp. 1344-1365, 2008.

[24] Q. Jiu, Y. Wang, and Z. Xin, "Stability of rarefaction waves to the 1D compressible Navier-Stokes equations with densitydependent viscosity," Communications in Partial Differential Equations, vol. 36, no. 4, pp. 602-634, 2011.

[25] Q. Jiu and Z. Xin, "The Cauchy problem for 1D compressible flows with density-dependent viscosity coefficients," Kinetic and Related Models, vol. 1, no. 2, pp. 313-330, 2008.

[26] H.-L. Li, J. Li, and Z. Xin, "Vanishing of vacuum states and blowup phenomena of the compressible Navier-Stokes equations," Communications in Mathematical Physics, vol. 281, no. 2, pp. 401-444, 2008.

[27] D. Hoff and J. Smoller, "Solutions in the large for certain nonlinear parabolic systems," Annales de l'Institut Henri Poincaré: Analyse Non Linéaire, vol. 2, no. 3, pp. 213-235, 1985. 


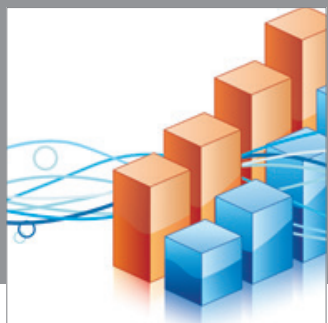

Advances in

Operations Research

mansans

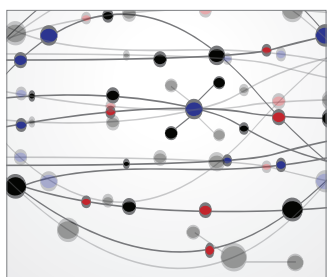

The Scientific World Journal
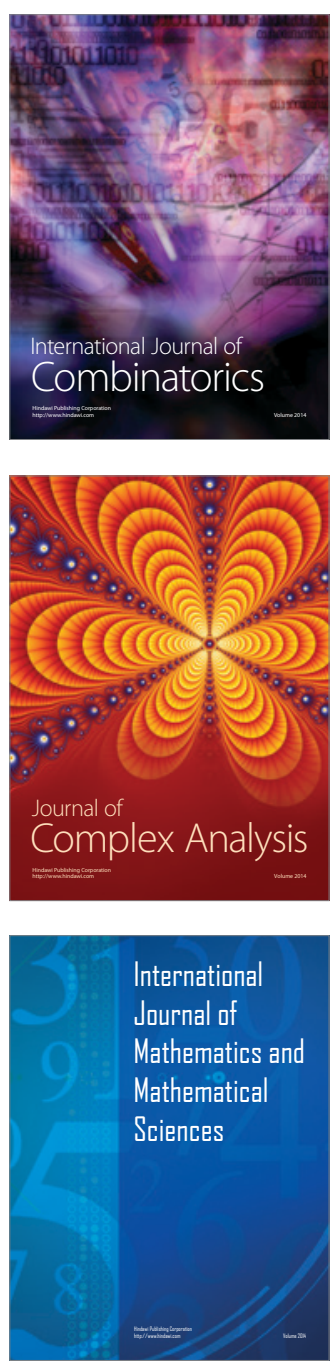
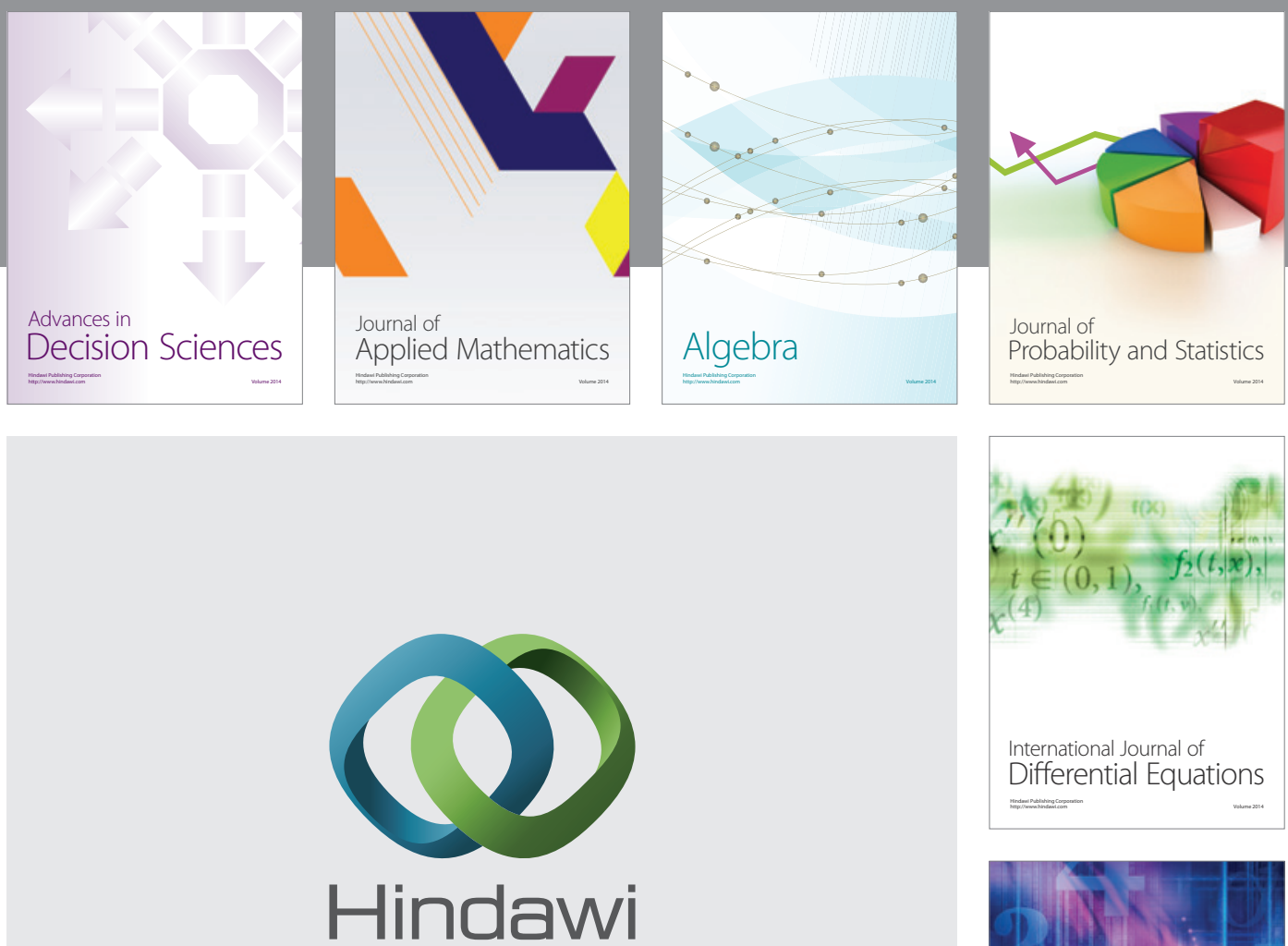

Submit your manuscripts at http://www.hindawi.com
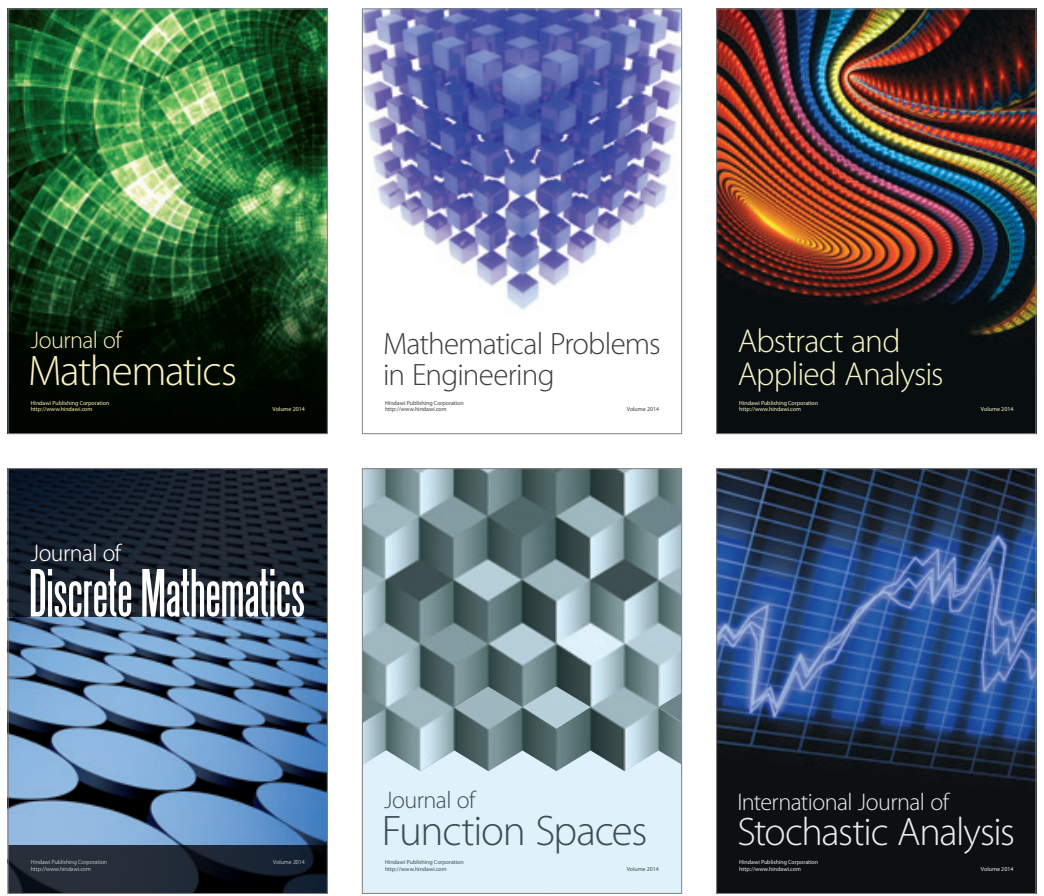

Journal of

Function Spaces

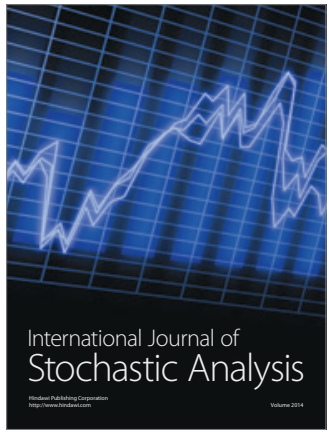

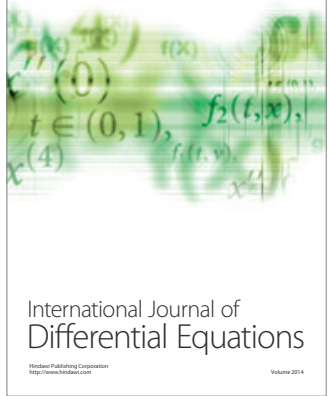
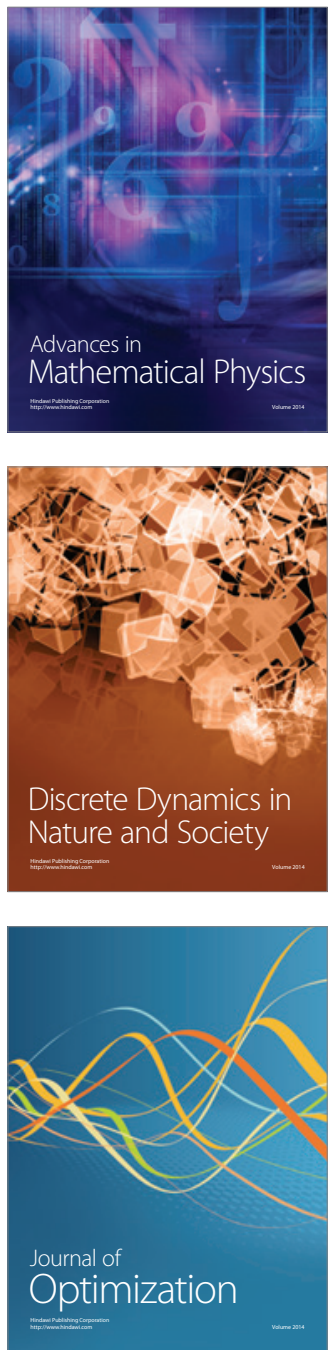